\title{
RADIATION EFFECTS ON MOS DEVICES: DOSIMETRY, ANNEALING, IRRADIATION SEQUENCE, AND SOURCES
}

\author{
E. G. Stassinopoulos* \\ G. J. Brucker** \\ O. Van Gunten*** \\ A. R. Knudson $\dagger$ \\ T. M. Jordan $\dagger \dagger$
}

ABSTRACT

This paper reports on some investigations of dosimetry, annealing, irradiation sequences, and radioactive sources, involved in the determination of radiation effects on MOS devices. Results show that agreement in the experimental and theoretical surface to average doses support the use of thermo-luminescent dosimeters (manganese activated calcium fluoride) in specifying the surface dose delivered to thin gate insulators of MOS devices. Annealing measurements indicate the existence of at least two energy levels, or activation energies, for recovery of soft oxide MOS devices after irradiation by electrons, protons, and gammas. Damage sensitivities of MOS devices were found to be independent of combinations and sequences of radiation type or energies. Comparison of various gamma sources indicated a small dependence of damage sensitivity on the Cobalt facility, but a more significant dependence in the case of the Cesium source. These results were attributed to differences in the spectral content of the several sources.

\section{Introduction}

When damage measurements are made on electronic devices at various Cobalt- 60 gamma ray facilities, the devices are usually surrounded by sufficient material (mostly aluminum) to assure electronic equilibrium. The damage is then stated as a function of the (Cobalt-60) dose they have received. When the devices operate onboard spacecraft, it is found that they usually exceed their predicted lifetimes, which are normally based on their Cobalt- 60 characterizations. The space environment contains principally protons and electrons. Previous work on the charge yields in MOS oxides irradiated by alpha particles, protons, and electrons $s^{1,2,3}$ have indicated a possible reason for this discrepancy, showing that the charge yields are different for different particle types and energies. However, these measurements are not directly applicable because MOS capacitors were used in that work and they have been found to give results which do not correlate quantitatively with those obtained from inverters. ${ }^{4}$ Recently, inverter measurements made on a set of well-characterized RCA CD4007 parts, all processed from a single wafer, have shown that damage in MOS devices depends strongly on the particle type, incident angle, energy, and on the operating conditions of the devices. ${ }^{5}$

During that study several issues concerning dosimetry, annealing, particle irradiation sequence, and radiation source effects were confronted and their impact on the principal objectives of the study were investigated. This paper reports on our findings from these investigations.

\footnotetext{
Manuscript received October 22, 1982; revised February 10, 1983.

* NASA/Goddard Space Flight Center, Greenbelt, MD

** RCA David Sarnoff Research Center, Princeton, NJ

*** National Security Agency, Fort Meade, MD

$\dagger$ Naval Research Laboratory, Washington, DC

$\dagger \dagger$ Experimental and Mathematical Physics Consultants, Simi Valley, CA
}

Results

\section{Dosimetry}

In all tests to be discussed, the lids of the irradiated MOS devices were removed prior to exposure. Manganese activated calcium fluoride TLD dosimetry $\left(\mathrm{CaF}_{2}: \mathrm{Mn}\right)$ was used for the electron and proton irradiations as described in reference (5). However, the particle doses were not uniform throughout the dosimeters or in the CMOS gate structure, but had a rather complex depth-dose profile. 6,7 Great care was exercised to insure that this non-uniformity was properly accounted for by comparing responses of TLDs of $0.0254,0.254$, and 0.889 $\mathrm{mm}$ thicknesses $(1,10$, and $35 \mathrm{mil}$, respectively) with theoretical and experimental depth-dose curves for electrons and theoretical curves for protons in $\mathrm{CaF}_{2}: \mathrm{Mn}$.

This was done because the luminescent readout from the TLD's did not correspond to the surface doses of the incident particles, but to the total energy deposited in the TLD, as they were slowed down in their transit through the dosimeter. The integral of this energy depends upon the dosimeter thickness and the energy of the incident primary particles. To obtain the MOS gate dielectric dose, the relationship between the TLD readout and the surface dose must be established.

Therefore, electron depth-dose profiles in manganese activated calcium fluoride were approximated by using data (range and stopping power) for aluminum and copper with appropriate weighting factors ${ }^{7}$, as illustrated in Figure 1. These two elements bracket the effective atomic number and density of $\mathrm{CaF}_{2}: \mathrm{Mn}$. The resultant depth-dose curves were then integrated to determine the normalized average dose throughout the dosimeter. The ratio between the surface dose and this average dose was then calculated for each electron energy and TLD thickness combination. The results are shown in Table 1a.

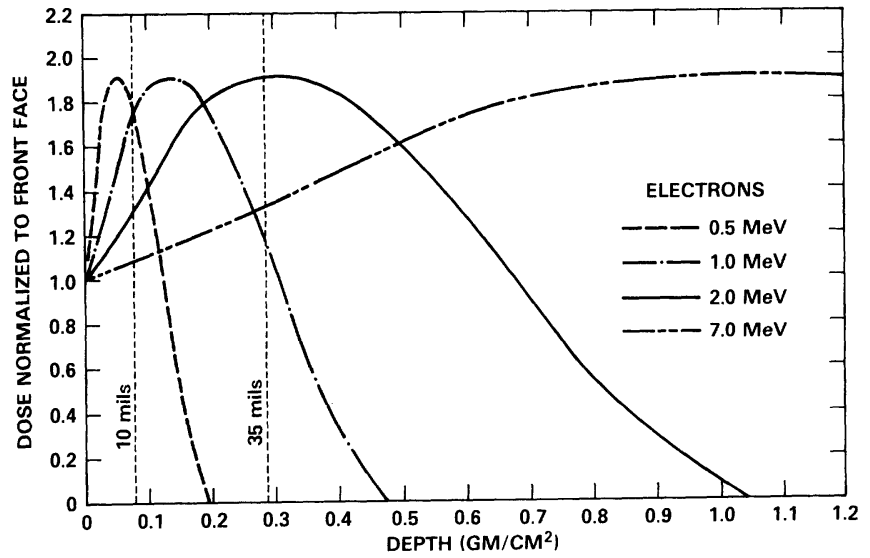

Figure 1. Electron Depth-Dose Profiles in Manganese Activated Calcium Fluoride TLD's.

This ratio could then be applied as a correction factor to the TLD readout to determine the most probable surface dose and thereby the dose in the CMOS gate dielectric. 
Table 1a. Comparison of Surface Dose to Average Dose

\begin{tabular}{ccc}
\hline $\begin{array}{c}\text { Electron } \\
\text { Energy }\end{array}$ & $\begin{array}{c}\text { TLD } \\
\text { Thickness }\end{array}$ & $\begin{array}{c}\text { Calculated Surface to } \\
\text { Average Dose Ratio }\end{array}$ \\
\hline $.5 \mathrm{MeV}$ & $0.254 \mathrm{~mm}(10 \mathrm{mil})$ & 0.60 \\
& $0.889 \mathrm{~mm}(35 \mathrm{mil})$ & 1.29 \\
$1.0 \mathrm{MeV}$ & $0.254 \mathrm{~mm}(10 \mathrm{mil})$ & 0.71 \\
& $0.889 \mathrm{~mm}(35 \mathrm{mil})$ & 0.62 \\
$2.0 \mathrm{MeV}$ & $0.254 \mathrm{~mm}(10 \mathrm{mil})$ & 0.85 \\
& $0.889 \mathrm{~mm}(35 \mathrm{mil})$ & 0.64 \\
$7.0 \mathrm{MeV}$ & $0.254 \mathrm{~mm}(10 \mathrm{mil})$ & 0.95 \\
& $0.889 \mathrm{~mm}(35 \mathrm{mil})$ & 0.86 \\
\hline
\end{tabular}

A crosscheck on this technique for determining surface dose was conducted by performing a series of measurements where two dosimeter thicknesses were overlayed and simultaneously irradiated with an equal fluence of electrons. This involved TLD pairs of 0.0254 to $0.254 \mathrm{~mm}$ ( 1 to 10 mils) and 0.0254 to $0.889 \mathrm{~mm}$ ( 1 to $35 \mathrm{mils}$ ). While there was a considerable difference in the dose profiles for $0.254 \mathrm{~mm}(10 \mathrm{mil})$ versus $0.889 \mathrm{~mm}(35 \mathrm{mil})$ dosimeters and therefore a corresponding difference in their readouts, the surface dose for both dosimeters had to be equal, and thus the calculations should have yielded equal values for these two cases. The results are listed in Table $1 \mathrm{~b}$ where the 0.0254 to $0.254 \mathrm{~mm}$ and 0.0254 to 0.889 TLD measurements ( 1 to 10 and 1 to 35 mil, respectively) are compared in column three and the similar values of Table 1a are repeated in column four for convenience of comparison. The agreement of the results of these two approaches was good. In addition to these checks, theoretical dose-depth curves for electrons in $\mathrm{CaF}_{2}: \mathrm{Mn}$ were calculated, as shown in Figure 2, using theoretical electron transport codes. ${ }^{8}$ Surface-to-average dose ratios were then determined from the curves of Figure 2. These values are listed in column five of Table $1 \mathrm{~b}$.

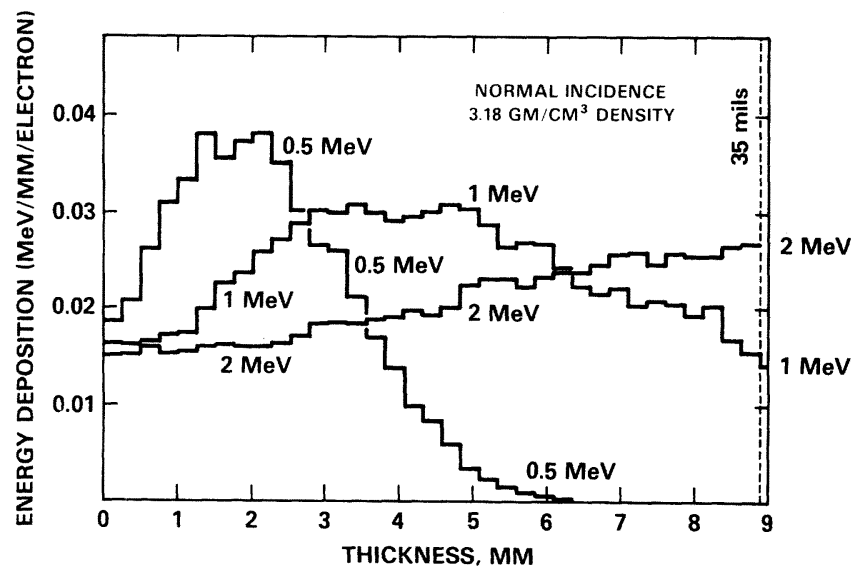

Figure 2. Electron Energy Deposition Curves in Manganese Activated Calcium Fluoride.

Table 1b. Comparison of Surface Dose to Average Dose Determined irom Ratios of $0.889 \mathrm{~mm}(35 \mathrm{mil})$ and $0.254 \mathrm{~mm}(10 \mathrm{mil})$ TLD Doses to $0.0254 \mathrm{~mm}$ (1 mil) TLD Doses.

\begin{tabular}{|c|c|c|c|c|c|}
\hline \multirow{2}{*}{$\begin{array}{l}\text { Electron } \\
\text { Energy }\end{array}$} & \multicolumn{2}{|c|}{ TLD Pairs } & \multirow{2}{*}{$\begin{array}{c}\text { Experimental } \\
\text { Dose Ratio }\end{array}$} & \multirow{2}{*}{$\begin{array}{l}\text { Calculated Dose } \\
\text { Ratio from } \\
\text { Table 1a }\end{array}$} & \multirow[b]{2}{*}{$\begin{array}{l}\text { Theoretical } \\
\text { Dose Ratio }\end{array}$} \\
\hline & $\mathrm{mm}$ & mil & & & \\
\hline \multirow[t]{2}{*}{$1.0 \mathrm{MeV}$} & $.0254 / .254$ & $1 / 10$ & 0.74 & 0.71 & 0.79 \\
\hline & $.0254 / .889$ & $1 / 35$ & 0.83 & 0.62 & 0.67 \\
\hline \multirow[t]{2}{*}{$2.0 \mathrm{MeV}$} & $.0254 / .254$ & $1 / 10$ & 0.95 & 0.85 & 0.96 \\
\hline & $.0254 / .889$ & $1 / 35$ & 0.65 & 0.64 & 0.73 \\
\hline
\end{tabular}

A similar set of theoretical depth-dose curves for protons in manganese activated calcium fluoride were also calculated using proton transport $\operatorname{codes}^{8}$, and the surface-to-average dose ratios were then determined for the various experimental proton energies. Figure 3 illustrates these curves. Table 2 lists the ratios, as used to adjust the TLD average dose readings to the required surface doses.

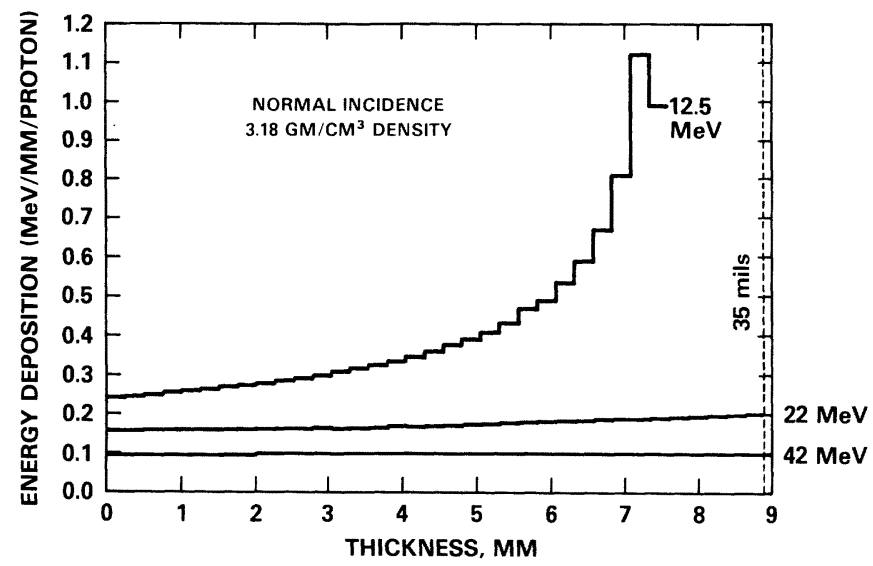

Figure 3. Proton Energy Deposition Curves in Manganese Activated Calcium Fluoride.

Table 2. Surface-to-Average Dose Ratios Determined from Theoretical Depth-Dose Curves for Protons in $0.889 \mathrm{~mm}(35 \mathrm{~mm})$ Manganese Activated Calcium Fluoride.

\begin{tabular}{cc}
\hline $\begin{array}{c}\text { Proton Energy } \\
(\mathrm{MeV})\end{array}$ & $\begin{array}{c}\text { Theoretical Dose } \\
\text { Ratio }\end{array}$ \\
\hline 12.3 & 0.61 \\
22.0 & 0.90 \\
42.0 & 0.98 \\
\hline
\end{tabular}

The electron and proton energy deposition profiles for $\mathrm{CaF}_{2}: \mathrm{Mn}$ were obtained from Monte Carlo calculations. Electron transport models included angular and energy loss straggling. The continuous slowing down approximation was used for proton transport. Calculated data points $(0.0254 \mathrm{~mm}=1 \mathrm{mil}$ layers $)$ had one sigma standard deviations of $\sim 5 \%$ for electrons and less than $1 \%$ for protons.

\section{Annealing}

After each series of exposures described in references (5) and (9), test samples were placed in sockets which applied bias to the devices under the same configurations as during exposure. The recovery or annealing of the samples was monitored as a function of time. It was determined, that device annealing was insignificant during any series of exposures within the time needed to take data $(\sim 1$ hour $)$, as illustrated in Figure 4 where the percentage recovery of $\Delta \mathrm{V}_{\mathrm{TN}}$ relative to the post-rad voltage shift is plotted versus logarithmic value of annealing time. During annealing, the exposure bias conditions were maintained for at least $\mathrm{T}_{\mathrm{A}} \simeq 2700$ hours on most devices. 


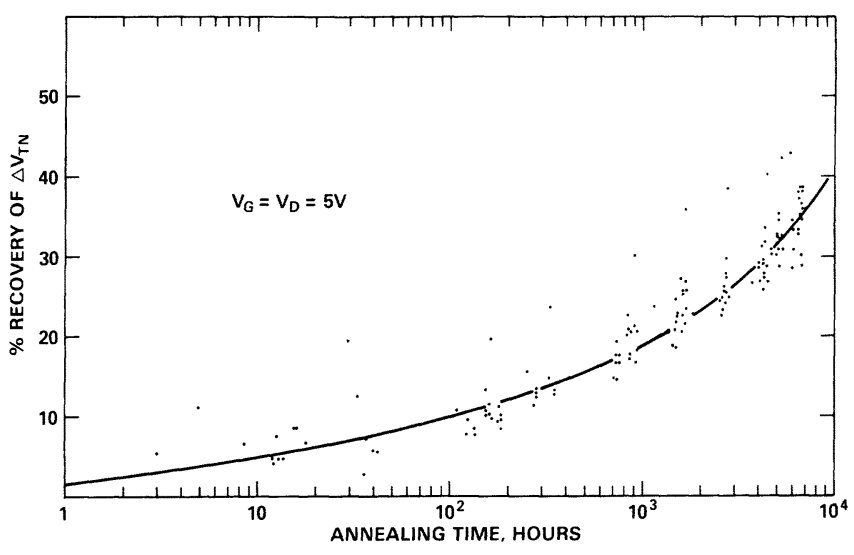

Figure 4. Annealing of Devices Exposed to Combinations of Electron, Proton, and Gamma Irradiations.

These data represent all of the test samples used in the experiment ${ }^{5}$, which were repeatedly exposed to combinations of electrons, protons, and gammas, with input gates of the inverter in the high state (i.e. $\mathrm{V}_{\mathrm{G}}=\mathrm{D}_{\mathrm{D}}$ ). The scatter in the data in spite of the damage normalization indicated the possibility of particle type and energy dependence. In Figure 5 a similar plot is shown for samples which were irradiated by only a single particle type. It can be seen that there appears to be a difference in the recovery rates of electron or gamma irradiated samples versus proton irradiated samples. In addition, there appear to be two distinct recovery time constants, namely, a slow one in the early stage of recovery and a faster one during the late stage. The inception or starting time of this late stage also appears to depend on particle type and energy. A further interesting result is the distinctly different recovery observed for the device irradiated with several electron energies (curve marked by $\mathrm{X}^{p} \mathrm{~s}$ ).

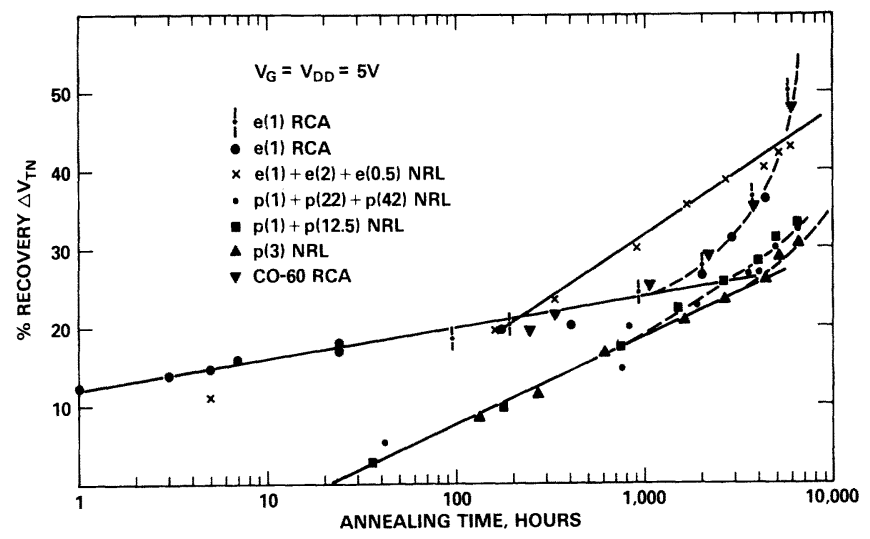

Figure 5. Annealing of Devices Exposed to Discrete Single Source Electron, Proton, and Gamma Irradiations.

An auxilary experiment, which was carried out with electrons to obtain the data in Tables $1 \mathrm{a}$ and $1 \mathrm{~b}$, provided additional samples which had been exposed to only one particle type. Figure 6 is a plot of percentage voltage recovery versus logarithmic value of annealing time $\mathrm{T}_{\mathrm{A}}$ for these samples. It can be seen that the characteristic slow and fast annealing time constants were reproduced by these electron irradiated samples. It should be noted that all of the samples were allowed to anneal at room temperature, but this was not controlled. Also, the ambient temperature during transportation could have varied by up to $\sim 40^{\circ} \mathrm{C}$.

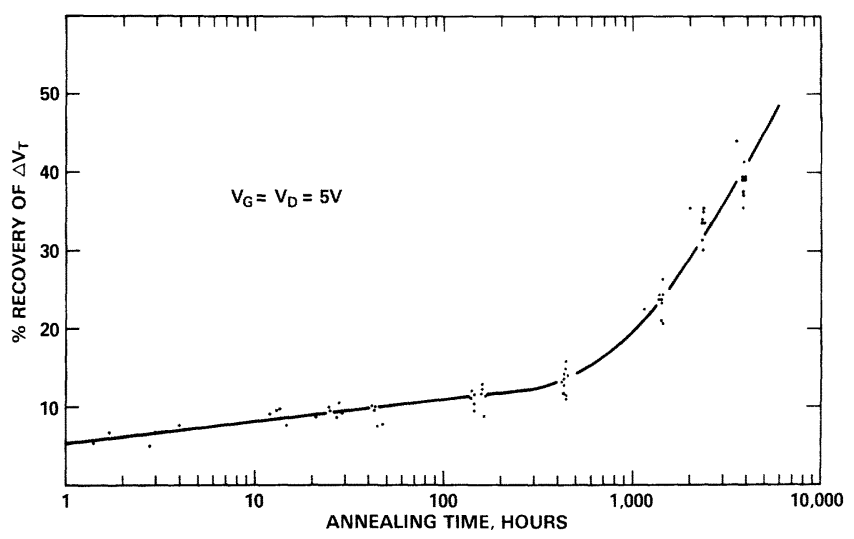

Figure 6. Annealing of Devices Exposed to Separate Electron Irradiations.

\section{Particle Irradiation Sequences}

During the early planning stages for the damage equivalence experiment ${ }^{10}$ it was assumed that the sequence in which a given test sample was exposed to different radiation sources might affect the results; consequently, data were taken in all possible combinations of sources and energies. However, comparison of damage sensitivities, $\Delta \mathrm{V}_{\mathrm{TN}} / \mathrm{DOSE}$, for each particle type and energy, indicated that the results are independent of sequence and particle combination. For example, grouping the sample data without regard to sequence or combination yielded percentage standard deviations for each particle type and energy which only ranged from 4.7 to $10.7 \%$.

\section{Radiation Sources}

The test samples also offered an opportunity to compare different cobalt sources and their spectral content, as well as a cesium source, by measuring the damage sensitivity at the various facilities. RCA has a Gamma Cell 220 which consists of a cylindrical radiation chamber surrounded by $8^{\prime \prime}$ pencils of Cobalt- 60 and these in turn are surrounded by heavy lead walls. This type of source is used by most industrial firms. The Fort Monmouth Cobalt- 60 facility consists of a point capsule type source, located in a shielded room. The NRL cobalt source was a point capsule type source located in a small lead container with a conical exit port which is about the same geometry as the Fort Monmouth cesium source. This latter source is used by the Army for calibration of radiation monitors for field use, and is called a UDM1A. In all cobalt and cesium tests, the sample devices were shielded by aluminum thick enough to provide electronic equilibrium. Table 3 contains the results of our comparison of damage sensitivities for the three cobalt and one cesium facilities. For all cobalt sources a $1 \%$ accuracy in calibration has been quoted.

Table 3. Comparison of Damage Sensitivities $\Delta \mathrm{V}_{\mathrm{TN}} /$ DOSE Measured by Devices with $\mathrm{V}_{\mathrm{DD}}=\mathrm{V}_{\mathrm{G}}=5 \mathrm{~V}$ During Exposure at the Various Facilities.

\begin{tabular}{ccccc}
\hline Facility & $\begin{array}{c}\text { Type } \\
\text { of Source }\end{array}$ & $\begin{array}{c}\text { \# of Data } \\
\text { Points } \\
\text { (measurements) }\end{array}$ & $\begin{array}{c}\text { Average } \\
\Delta V_{\text {TN }} / \text { Dose, } \\
\text { (Volts/Krad) }\end{array}$ & $\begin{array}{c}\% \text { Differ. } \\
\text { Relative } \\
\text { to NRL }\end{array}$ \\
\hline NRL & Co-60 & 13 & $.163 \pm 5 \%$ & $0 \%$ \\
RCA & Co-60 & 10 & $.143 \pm 4 \%$ & $12 \%$ \\
Ft. Monm. & Co-60 & 10 & $.142 \pm 4 \%$ & $13 \%$ \\
& Cesium-137 & 10 & $.113 \pm 7 \%$ & $31 \%$ \\
\hline
\end{tabular}




\section{Discussion}

Figure 1 illustrated that the doses from mono-energetic electrons normally incident upon a semi-infinite surface rise from the surface dose value to a maximum value approximately $80 \%$ greater. This peak value then falls off to zero at a depth slightly greater than the maximum electron range in the material. The gate oxide is only $1100 \AA$ thick and covered by about 1 to 2 micrometers of aluminum and silicon dioxide; thus there were no significant discontinuities of atomic number $(\mathrm{z})$ in this region of the structure. The doses in the gate dielectric should be equal to the surface doses.

The agreement between the experimental surface-to-average dose ratios, the theoretical values, and the experimental ratios from the TLD comparisons (see Table la and $1 \mathrm{~b}$ ) provided confidence in the dosimetry of the damage equivalence experiment of reference (5). Corrections to the electron damage sensitivities, $\Delta \mathrm{V}_{\mathrm{TN}} / \mathrm{DOSE}$, were based on the experimental TLD comparisons. These data were judged as the best representative values of actual experimental conditions, as for example, accurate TLD composition and thickness.

The recovery characteristics at room temperature, illustrated by Figures 5 and 6 , show a dependence of annealing on radiation type during the early stages of recovery. It appears that for the late stage, the second or faster recovery rate occurred for all types of irradiated samples. There are evidently two distinct rates of recovery or time constants, namely, a slow rate followed by a faster recovery rate after the late stage. The work of reference (11) indicated that recovery of damage in inverter samples of the same type as involved in this study are governed by at least two activation energies. It is believed that the data of Figures 4, 5, and 6 can be explained as due to two defect levels and activation energies, as in reference (11). The shallow energy level was responsible for the early stage of recovery whereas the deeper level was responsible for the later stage. It also appears from the data of Figure 5, that the initial starting time for the second stage of recovery was different for the samples irradiated by protons of several energies compared to the samples irradiated by $1 \mathrm{MeV}$ electrons and gammas, and both of these sample groups differed from the sample irradiated by electrons of several energies. These data seem to indicate a dependence of activation energy of this deeper defect level on type of radiation and energy, and thus, the slower recovery due to these deeper energy levels during the early stages, compared to the electron or gamma irradiated samples. The apparent dependence of recovery on irradiation particles does not have any other detailed explanation at this time. Another feature of the annealing data was the independence of the late stage of recovery on maintenance of bias during recovery. The samples of Figures 4 and 5 were maintained under bias $\left(V_{G}=V_{D}=5\right.$ Volts) for $T_{A} \simeq 2700$ hours, then bias was removed and all leads shorted together. It can be seen in Figure 5 that the data points following the 2700 hours plotted on the same annealing curve as before. These results indicate that annealing is occurring without the help of bias induced electron injection from silicon to oxide.

The test device used in these experiments turned out to be a very sensitive and repeatable sensor for comparing the spectra of different radioactive sources. Previous work in the literature ${ }^{12}$ has indicated that the spectral content of gammas from a supposedly monoenergetic source can impact device characterization data due to scattering in the source and surroundings. Table 3 lists the results of a comparison study of several sources. The geometries of the sources were quite different. The NRL point source appears to have the simplest geometry and was expected to supply the hardest spectra. The Fort Monmouth source was an approximate point source and it was raised by air pressure in a steel pipe outside the container; however, $45.7 \mathrm{~cm}$ (1-1/2 feet) behind the pipe was a wall and a lead shield. It can be seen from Table 3 that the RCA and Fort Monmouth sources yielded lower damage compared to the NRL source. The cesium source emits a $.66-\mathrm{MeV}$ gamma ray, and it was expected that the greater scattering of the lower energy gammas would produce the softest spectrum.
The Table 3 data indicate that this was indeed the case with a $31 \%$ lower damage sensitivity.

\section{Conclusions}

The agreement in the experimental and theoretical ratios of surface to-average doses support the use of TLDs in MOS device tests, but at the same time the derived adjustments to the TLD doses illustrate the importance of specifying the surface dose delivered to the oxide layer by particulate radiation. The annealing results indicate the existence of at least two energy levels or activation energies for recovery of soft oxide MOS devices at room temperature under bias. There also appears to be a difference in the early stages of recovery for samples irradiated solely by protons as compared to electrons or gamma rays. The proton irradiated samples recovered at a slower rate in the early stage of annealing. The inception of the later stage of recovery also appears to be dependent on particle type and energy.

Results show that damage sensitivities of MOS devices were independent of combinations and sequences of particle types (electrons, protons, and gammas) or energies. There appear to be small differences in damage sensitivities measured with devices exposed to different Cobalt- 60 facilities and larger differences when devices were exposed to a Cesium source. These differences can be due to variations in the spectral contents of the several sources.

\section{Acknowledgements}

The authors wish to thank Jim Ritter of the Naval Research Laboratory, Jim Vette of NASA-Goddard Space Flight Center, and Jim McGarrity of the Harry Diamond Laboratories for many helpful and valuable discussions and suggestions.

\section{References}

1. T. R. Oldham and J. M. McGarrity, "Ionization of $\mathrm{SiO}_{2}$ by Heavy Charged Particles", IEEE Trans. Nucl. Sci., NS-28, No. 6, Dec. 1981.

2. H. E. Boesch, Jr. and J. M. McGarrity, "Charge Yield and Dose Effects In MOS Capacitors at $80^{\circ} \mathrm{K}$ ", IEEE Trans. Nucl. Sci., NS-23, No. 6, Dec. 1976.

3. O. L. Curtis, Jr., J. R. Srour, and K. Y. Chiu, "Hole and Electron Transport in $\mathrm{SiO}_{2}$ Films", J. Appl. Phys., 45, 4506, 1974.

4. T. W. Griswold and J. Maserjian, "Test Chips in Reliability Assurance", GOMAC, Digest of Papers, 398, Nov. 1978.

5. G. J. Brucker, E. G. Stassinopoulos, O. Van Gunten, L. S. August and T. M. Jordan, "The Damage Equivalence of Electrons, Protons, and Gamma Rays in MOS Devices", IEEE Trans. Nucl. Sci., NS-29, No. 6, Dec. 1982.

6. S. E. Chappell and J. C. Humphreys, "Silicon Detector Measurments of Energy Desposition in Aluminum by Monoenergetic Electrons", IEEE Trans. Nucl. Sci., NS-17, No. 6, Dec. 1970.

7. Harvey Eisen, "Electron Depth Dose Distribution Measurements in Metals and Two Slab Layers", Doctoral Thesis, University of Maryland, 1971.

8. T. M. Jordan, "NOVICE: A Radiation Transport and Shielding Code", EMP.L82.001, January 1982. 
9. G. J. Brucker, E. G. Stassinopoulos, J. C. Ritter, O. Van Gunten, L. S. August, A. B. Campbell, E. L. Petersen, P. Shapiro, A. R. Knudsen, L. T. Myers, and J. D. Mann, "The Damage Equivalence Experiment of Electrons, Protons and Gamma Rays in MOS Devices", NASA Goddard X-601-81-36, Nov. 1981.

10. E. G. Stassinopoulos and G. J. Brucker, "An Experiment to Determine Damage Equivalence of Electrons, Protons, and Gamma Rays in MOS Devices", NASA Goddard X-601-81-37, Oct. 1980.
11. V. Danchenko, E. G. Stassinopoulos, P. H. Fang, and S. S. Brashears, "Activation Energies of Thermal Annealing of RadiationInduced Damage in n- and p-channels of CMOS Integrated Circuits", IEEE Trans. Nucl. Sci., NS-27, No. 6, December 1980.

12. E. A. Burke. J. J. Boyle, and H. J. Huemmler, "Gamma-Induced Noise in CCDs", IEEE Trans. Nucl. Sci., NS-28, No. 6, Dec. 1981. 